\title{
A Convex-Risk-Measure Based Model and Genetic Algorithm for Portfolio Selection
}

\author{
Weijia Wang, ${ }^{1}$ Jie Hu, ${ }^{1}$ and Ning Dong ${ }^{2,3}$ \\ ${ }^{1}$ International Business School, Shaanxi Normal University, Xi'an 710119, China \\ ${ }^{2}$ School of Mathematics and Statistics, Xidian University, Xian 710071, China \\ ${ }^{3}$ School of Mathematics and Information Science, Shaanxi Normal University, Xian 710119, China
}

Correspondence should be addressed to Weijia Wang; weijiawang03@hotmail.com

Received 19 August 2014; Accepted 8 October 2014

Academic Editor: Yuping Wang

Copyright (C) 2015 Weijia Wang et al. This is an open access article distributed under the Creative Commons Attribution License, which permits unrestricted use, distribution, and reproduction in any medium, provided the original work is properly cited.

\begin{abstract}
A convex risk measure called weighted expected shortfall (briefly denoted as WES (Chen and Yang, 2011)) is adopted as the risk measure. This measure can reflect the reasonable risk in the stock markets. Then a portfolio optimization model based on this risk measure is set up. Furthermore, a genetic algorithm is proposed for this portfolio optimization model. At last, simulations are made on randomly chosen ten stocks for 60 days (during January 2, 2014 to April 2, 2014) from Wind database (CFD) in Shenzhen Stock Exchange, and the results indicate that the proposed model is reasonable and the proposed algorithm is effective.
\end{abstract}

\section{Introduction}

In 1952, Markowitz proposed the first quantitative risk measure (i.e., variance) for portfolio selection [1]. Because variance as the risk measure has essential drawbacks such as only being applicable to the cases in which the return obeys the normal distribution or elliptical distribution, it is rarely used as the risk measure currently. After that, value at risk (VaR) was proposed by Baumol [2] as a risk measure and it has been a widely used risk measure to manage or control risk for portfolio selection. For example, the Basle Committee on Banking Supervision allows banks to use VaR when determining their asset-adequacy requirements arising from their exposure to market risk [3]. The reason for VaR to be a popular risk measure is that it is easily understood and can exactly answer the following question: under the normal market environment and given confidence level, what is the maximal potential loss of an investor in a certain period? Just as $\mathrm{VaR}$ definition mentioned, given some confidence level $\alpha \in(0,1)$, the VaR of the portfolio at the confidence level $\alpha$ is given by the smallest number $l$ such that the probability of the loss $L$ exceeding $l$ is not larger than $(1-\alpha)$; that is,

$$
\operatorname{VaR}_{\alpha}=\inf \{l \in R \mid P[L>l] \leq 1-\alpha\}
$$

Since VaR appears, many researchers have paid attention to use VaR in their portfolio models. For example, authors in [4] integrate GARCH model and VaR model and got the better results. However, VaR model has some serious drawbacks: (1) it usually gives preserved estimation on the risk of the markets [5]; (2) it does not satisfy subadditivity (i.e., the total risk of the sum of two investments is not larger than that of the sum of their risks [6]); and (3) it is not a coherent risk measure [7]. Thus, some researchers thought that VaR was not a good risk measure and even some researchers thought that it was seductive but dangerous (e.g., [8]). To get more reasonable risk measures, researchers discussed the conditions which a reasonable risk measure should satisfy (e.g., $[6,7,9,10])$. The widely accepted and representative condition for a reasonable risk measure is coherent condition, which defines a risk measure $\rho(x)$ to be a coherent measure if it satisfies the following properties:

(I) monotonity: if $x \leq y, \rho(x) \geq \rho(y)$, for any two portfolios $x$ and $y$;

(II) subadditivity: $\rho(x+y) \leq \rho(x)+\rho(y)$, for any two portfolios $x$ and $y$;

(III) positive homogeneity: $\rho(\theta x)=\theta \rho(x)$, for any portfolio $x$, and $\theta \geq 0$; 
(IV) translational invariance: If $A$ is a deterministic portfolio with guaranteed return $a$, then $\rho(x+a)=\rho(x)-a$ for any portfolio $x$.

Monotonity illustrates that if portfolio y always has better values than portfolio $\mathrm{x}$ under almost all scenarios, then the risk of $y$ should be less than the risk of $x$. Subadditivity implies that the risk of two portfolios together cannot get any worse than adding the two risks separately; this is the diversification principle. Loosely speaking, positive homogeneity represents that if you double your portfolio then you will double your risk. Translational invariance indicates that after adding an amount of money $A$ to the original portfolio $x$, the risk will be decreased by amount $a$.

Based on concept of coherent risk measure, many new risk measures were proposed. For examples, Artzner et al. proposed a coherent risk measure WEC $\alpha$ [7]. WEC $\alpha$ is a good risk measure in theory, but it seriously depends on the distribution of random variable $x$. To get easily computed coherent risk measure, Acerbi et al. [11] proposed the expected shortfall as the risk measure (ES $\alpha$ ) and Rockafellar and Uryasev [12] proposed a coherent risk measure: conditional value at risk (CVaR). Although the definitions of $\mathrm{ES} \alpha$ and $\mathrm{CVaR}$ are different, their key ideas are same. ES $\alpha$ has an important property [13]: any coherent risk measure can be described by spectral risk measures and any coherent spectral risk measure can be represented by the linear combination of $\mathrm{ES} \alpha$ with different confidence levels. That means that ES $\alpha$ is the basic components of coherent risk measures. CVaR also has attractive properties such as its formula can be easily obtained and thus can be easily used for portfolio selection. Recently, with the further deep study of coherent risk measures, some new coherent risk measures with good performance were proposed; for example, Rosazza Gianin [14] proposed a coherent risk measure based on g-expectation operator which can be applicable not only to the estimation of financial asserts, but also to the derivatives. Also, by choosing different g-expectation operator, this risk measure can satisfy the different prefers of investors to the risk. To handle the problems with nonnormal distribution and leptokurtosis (i.e., fat tails), Chen and Wang [15] proposed a new class of coherent risk measures based on $p$-norms. However, aforementioned coherent risk measures are only one side coherent risk measures [16]; that is, the distribution information on only one side of supply side and demand side is considered. From the point of view of competition, when an investor is going to buy a stock, he or she should also pay attention to the behavior of the seller. That is to say, to measure the risk more exactly, the behavior of investors to supply side and demand side should be simultaneously considered. In other words, two-side risk measures are needed. For example, Chen and Wang [16] proposed a two-side coherent risk measure which is easily applied.

Although there have been a lot of progresses in the research of coherent risk measures, there exist two serious problems in coherent risk measures. (1) Positive homogeneity is not reasonable in many cases and the condition is too strong $[17,18]$; (2) translational invariance is not reasonable in many cases and more and more researchers do not adopt this condition in their risk measures (e.g., [19-21]). To relax the condition in positive homogeneity and get more reasonable risk measures, some authors suggested replacing subadditivity and positive homogeneity by the following convexity:

$$
\rho(\lambda x+(1-\lambda) y) \leq \lambda \rho(x)+(1-\lambda) \rho(y), \quad \forall \lambda \in[0,1],
$$

and proposed convex risk measures (e.g., [22, 23]). From above analysis, one can see that a good and reasonable risk measure should satisfy monotonity and convexity. For this purpose, some risk measures satisfying monotonity and convexity were proposed (e.g., [24, 25]) and experiments indicate that the measures are reasonable.

In this paper, we adopt a convex risk measure called weighted expected shortfall (WES) as the risk measure [24] and propose a portfolio optimization model based on this risk measure. Then we design a genetic algorithm for this portfolio optimization model. At last, simulations are made on real data in the financial markets and the results indicate that the proposed model is reasonable and the proposed algorithm is effective.

The remaining parts are organized as follows. In Section 2, the portfolio optimization model is set up. The proposed genetic algorithm is proposed in Section 3. The computer simulations are made on real data in financial markets in Section 4, and the conclusions are made in Section 5.

\section{Portfolio Optimization Model}

In static state, risk can be seen as a random variable $X$ on a probability space $(\Omega, \mathscr{F}, P)$, where $X$ represents uncertain rate of return of portfolio. Then, for a given confidence level $\alpha$, a new risk measure is defined in $[24,26]$ as follows.

Definition 1 (see $[24,26]$ ). Suppose that $E\left(X^{-}\right)<\infty$; for given confidence level $\alpha$, a new risk measure called weighted expected shortfall $\left(\mathrm{WES}_{\alpha}\right)$ is defined as follows:

$$
\begin{array}{r}
\operatorname{WES}_{\alpha}(X)=\alpha^{-1}\left[w\left(x_{\alpha}\right) x_{\alpha}\left(P\left\{X \leq x_{\alpha}\right\}-\alpha\right)\right. \\
\left.-E\left(w(X) X \operatorname{sign}\left(x_{\alpha}\right)\right)\right],
\end{array}
$$

where $w(x)$ is monotone nonincreasing function and positive convex when $x \leq 0$ and nonnegative concave when $x>0$. For example, when $x \leq 0, w(x)=\exp (-\lambda x)$, and $w(x)=0$ when $x>0$, where $\lambda$ represents the risk aversion coefficient,

$$
\begin{gathered}
x_{\alpha}=\inf \{x \in R \mid P(X \leq x) \geq \alpha\}, \\
X^{-}=-\min (X, 0), \\
\operatorname{sign}\left(x_{\alpha}\right)= \begin{cases}1, & X \leq x_{\alpha} \\
0, & X>x_{\alpha},\end{cases}
\end{gathered}
$$

and $P(A)$ represents the probability of random event $A$.

Also, in $[24,26]$, a computable formula (or an estimation) of $\mathrm{WES}_{\alpha}(X)$ was given. Suppose that there are $n$ risky assets 
and one risk-free asset and the portfolio for these assets is denoted by $y=\left(y_{1}, y_{2}, \ldots, y_{n}, y_{n+1}\right)$, where $\left(y_{1}, y_{2}, \ldots, y_{n}\right)$ is the portfolio for $n$ risky assets, respectively, and $y_{n+1}$ is the portfolio for the risk-free asset. Let $r_{i t}$ represent the rate of return of the $i$ th risky asset for $i=1 \sim n$ and the rate of return of the risk-free asset for $i=n+1$, respectively, in the period $t$ for $t=1 \sim M$. Let $r_{i}$ represent the rate of return of the $i$ th risky asset for $i=1 \sim n$ and the rate of return of the risk-free asset for $i=n+1$, respectively. It can be estimated by

$$
r_{i}=\frac{1}{M} \sum_{t=1}^{M} r_{i t}, \quad i=1 \sim n+1 .
$$

Let $d_{i t}$ represent the dividend yield of the $i$ th risky asset for $i=1 \sim n$ in the period $t$ for $t=1 \sim M$. Let $d_{i}$ represent the dividend yield of the $i$ th risky asset for $i=1 \sim n$. It can be estimated by

$$
d_{i}=\frac{1}{M} \sum_{t=1}^{M} d_{i t}, \quad i=1 \sim n .
$$

Let $y^{0}=\left(y_{1}^{0}, y_{2}^{0}, \ldots, y_{n}^{0}, y_{n+1}^{0}\right)$ be the initial portfolio, let $k_{i}$ be the per unit transaction cost of the $i$ th risky asset, let $t_{g}$ be the asset income marginal tax rate, let $t_{0}$ be ordinary marginal income tax rate, and let $r_{p}$ be the given target rate of return. Let

$$
\begin{gathered}
y_{i}^{\prime}=\left(y_{i}-y_{i}^{0}\right)^{+}=\max \left(y_{i}-y_{i}^{0}, 0\right), \quad i=1 \sim n, \\
y_{i}^{\prime \prime}=\left(y_{i}-y_{i}^{0}\right)^{-}=-\min \left(y_{i}-y_{i}^{0}, 0\right), \quad i=1 \sim n, \\
g(y)_{t}=\sum_{i=1}^{n}\left[\left(1-t_{g}\right) r_{i t}+\left(1-t_{0}\right) d_{i t}\right] y_{i} \\
+\left(1-t_{0}\right) d_{n+1} y_{n+1}-\sum_{i=1}^{n} k_{i}\left(y_{i}^{\prime}+y_{i}^{\prime \prime}\right) \\
R_{i}=\frac{1-t_{g}}{M \sum_{t=1}^{M} r_{i t}+\left(1-t_{0}\right) d_{i}}, \quad i=1 \sim n, \\
R_{n+1}=\left(1-t_{0}\right) r_{n+1}, \\
G(y)=\sum_{i=1}^{n+1} R_{i} y_{i}-\sum_{i=1}^{n} k_{i}\left(y_{i}^{\prime}+y_{i}^{\prime \prime}\right) .
\end{gathered}
$$

If we choose the proper confidence level $\alpha$ and parameter $M$ such that $(1-\alpha) M$ is an integer, then, according to [26], the risk based on the new measure $\mathrm{WES}_{\alpha}$ can be calculated by

$$
\operatorname{WES}_{\alpha}(y)=-\frac{1}{(1-\alpha) M} \sum_{m=1}^{(1-\alpha) M} w\left(g(y)_{(m)}\right) g(y)_{(m)},
$$

where $g(y)_{(1)}$ is the smallest element of $\left\{g(y)_{t} \mid t=1 \sim M\right\}$ and $g(y)_{(m)}$ is the $m$ th smallest element of $\left\{g(y)_{t} \mid t=1 \sim\right.$ $M\}$.
Also, according to [26], an optimization portfolio model can be formulated as

$\min \operatorname{WES}_{\alpha}(y)=-\frac{1}{(1-\alpha) M} \sum_{m=1}^{(1-\alpha) M} w\left(g(y)_{(m)}\right) g(y)_{(m)}$

s.t. $\quad G(y) \geq r_{p}$

$$
\begin{aligned}
& \sum_{i=1}^{n+1} y_{i}=1 \\
& y_{i}=y_{i}^{\prime}-y_{i}^{\prime \prime}+y_{i}^{0}, \quad i=1 \sim n \\
& y_{i}^{\prime} \geq 0, \quad y_{i}^{\prime \prime} \geq 0, \quad i=1 \sim n \\
& l_{i} \leq y_{i} \leq u_{i}, \quad i=1 \sim n \\
& y_{i} \geq 0, \quad i=1 \sim n+1 .
\end{aligned}
$$

Also, the condition $y_{i}^{\prime} y_{i}^{\prime \prime}=0$ is required to be satisfied in optimal solution in [26]. This is a nonlinear constraint, but this condition was not put in the model in order to make the model easily solved (although this is not reasonable). Thus the optimal solution obtained for the model (14)-(20) may not satisfy the condition $y_{i}^{\prime} y_{i}^{\prime \prime}=0$. This will result in the obtained solution being not a true optimal solution.

Note that $y_{i}(i=1 \sim n+1), y_{i}^{\prime}$, and $y_{i}^{\prime \prime}(i=1 \sim n)$ are variables in the above model; thus, there are total $(3 n+1)$ variables and the problem dimension is $(3 n+1)$. But when we carefully check this model, we can find that these variables are not independent. In fact, from formulas (7) and (8), it can be seen that $y_{i}^{\prime}$ and $y_{i}^{\prime \prime}$ are not independent to $y_{i}$ and can be completely presented by $y_{i}$. Thus, variables $y_{i}^{\prime}$ and $y_{i}^{\prime \prime}$ can be deleted for $i=1 \sim n$. Also note that when we delete variables $y_{i}^{\prime}$ and $y_{i}^{\prime \prime}, i=1 \sim n$, constraints (17) and (18) as well as $y_{i}^{\prime} y_{i}^{\prime \prime}=0$ will be automatically satisfied. Furthermore, in order to estimate $R_{i}$ in formula (10) more precisely, we can use a large number of historic data (i.e., a large number $M$ ) in formulas (5), (6), and (10), but too large $M$ will result in the increasing of computation of risk function $\mathrm{WES}_{\alpha}(y)$. In order to reduce the computation load, we have to choose a proper $M$. In this way, we can simplify the model (14)-(20).

In summary, we can modify the above model by deleting variables $y_{i}^{\prime}$ and $y_{i}^{\prime \prime}, i=1 \sim n$ and constraints (17) and (18) as well as using proper value of $M$ to set up a new simplified optimization portfolio model as follows:

$$
\begin{array}{ll}
\min & \operatorname{WES}_{\alpha}(y)=-\frac{1}{(1-\alpha) M} \sum_{m=1}^{(1-\alpha) M} w\left(g(y)_{(m)}\right) g(y)_{(m)} \\
\text { s.t. } & G(y) \geq r_{p} \\
& \sum_{i=1}^{n+1} y_{i}=1 \\
& l_{i} \leq y_{i} \leq u_{i}, \quad i=1 \sim n \\
& y_{i} \geq 0, \quad i=1 \sim n+1 .
\end{array}
$$


TABLE 1: Stock names and their stock symbols randomly chosen.

\begin{tabular}{lcccccccccc}
\hline & S1 & S2 & S3 & S4 & S5 & S6 & S7 & S8 & S9 & S10 \\
\hline Stock name & VKA & PAB & BLL & SZPRDA & CSGA & SH & SZHA & SVOTA & Konka A & SSIA \\
Stock symbol & 000002 & 000001 & 000008 & 000011 & 000012 & 000014 & 000020 & 000018 & 000016 & 000019 \\
\hline
\end{tabular}

This optimization model has only $(n+1)$ dimensions which are much lower than those of the original model (14)(20).

\section{A New Genetic Algorithm for the Portfolio Model}

For notation convenience, let $y=\left(y_{1}, y_{2}, \ldots, y_{n}, y_{n+1}\right)$ denote the vector of all variables. Then $y$ is an $(n+1)$ dimensional vector. For notation convenience, let $\bar{n}=(n+1)$ in this section.

The optimization portfolio model in previous section is a nonlinear optimization problem. It is very hard to get its global optimal solution using the traditional optimization methods. Genetic algorithms (briefly, GAs) are a new kind of intelligent optimization methods which are designed for these difficult optimization problems [27-29]. They exploit a set of potential solutions, named a population, and detect the optimal solution through cooperation and competition among individuals of the population. However, for GAs in global optimization, the major challenges are that an algorithm may be trapped in the local optima of the objective function and the convergent speed may be slow. These issues are particularly challenging when the dimension of the problem is high and there are numerous local optima. In order to improve the GAs, researchers have incorporated other techniques to enhance their performance. One important technique is to design more efficient crossover operators to enhance the local search ability of GAs [28, 29]. In this section, we first design an efficient crossover operator which can explore the search space efficiently. Then we design a mutation operator which can adaptively exploit the search space. Based on these, a new genetic algorithm is proposed.

3.1. Crossover Operator. In this section, the uniform design method [28-30] is used to design a new crossover operator. The main objective of uniform design is to sample a small set of points from a given set of points, such that the sampled points are uniformly scattered on the interested region. The crossover operator based on the uniform design is similar to a local search scheme; thus, it can effectively explore the search space. The detail is as follows.

$$
\text { Denote } C_{\bar{n}}=\left\{\left(x_{1}, x_{2}, \ldots, x_{\bar{n}}\right) \mid 0 \leq x_{1}, \ldots, x_{\bar{n}} \leq 1\right\} \text {, }
$$

and let $\theta$ denote the decimal part of the real number $\theta$. A widely used method to generate $q$ approximately uniformly distributed points in $C_{\bar{n}}$ is as follows [30], where $q$ is a positive integer. Let $p$ be a positive prime, satisfying $p \geq 2 \bar{n}+3$.

$$
\begin{gathered}
\text { Denote } \omega=\frac{\pi}{p}, \\
\left(\gamma_{1}, \ldots, \gamma_{\bar{n}}\right)=2(\cos (\omega), \ldots, \cos (\bar{n} \omega)),
\end{gathered}
$$

TABLE 2: Optimal portfolios and their WESs in different risk aversion coefficients.

\begin{tabular}{lccccc}
\hline$\lambda$ & 0 & 0.1 & 1 & 5 & 10 \\
\hline S1 & 0.1410 & 0.1405 & 0.1272 & 0.1249 & 0.1266 \\
\hline S2 & 0.0000 & 0 & 0.0104 & 0.0079 & 0.0082 \\
\hline S3 & 0.0080 & 0.0079 & 0.0384 & 0.0699 & 0.0833 \\
\hline S4 & 0.1103 & 0.1107 & 0.1058 & 0.1208 & 0.1287 \\
\hline S5 & 0.0500 & 0.0499 & 0.0261 & 0.0000 & 0 \\
\hline S6 & 0.0805 & 0.0808 & 0.0830 & 0.0750 & 0.0660 \\
\hline S7 & 0.0783 & 0.0771 & 0.0931 & 0.0869 & 0.0771 \\
\hline S8 & 0.1433 & 0.1431 & 0.1289 & 0.1278 & 0.1255 \\
\hline S9 & 0.0976 & 0.0983 & 0.1046 & 0.1163 & 0.1168 \\
\hline S10 & 0.2910 & 0.2918 & 0.2823 & 0.2704 & 0.2678 \\
\hline WES & 0.0252 & 0.0256 & 0.0294 & 0.0495 & 0.0933 \\
\hline
\end{tabular}

and then $\left\{k\left(\gamma_{1}, \gamma_{2}, \ldots, \gamma_{\bar{n}}\right) \mid k=1 \sim q\right\}$ is a set of $q$ approximately uniformly distributed points in $C_{\bar{n}}$.

Suppose that $X=\left(x_{1}, x_{2}, \ldots, x_{\bar{n}}\right)$ and $Y=$ $\left(y_{1}, y_{2}, \ldots, y_{\bar{n}}\right)$ are any two parents chosen for crossover. Let $l_{i}=\min \left\{x_{1}, y_{1}\right\}$ and $u_{i}=\max \left\{x_{1}, y_{i}\right\}$ for $i=1 \sim \bar{n}$. Now a new crossover operator is designed to generate $q$ approximately uniformly distributed points in the following set:

$$
[l, u]=\left\{x \in R^{\bar{n}} \mid l_{i} \leq x_{i} \leq u_{i}, \quad i=1 \sim \bar{n}\right\}
$$

Algorithm 2 (crossover operator). (1) Generate $q$ approximately uniformly distributed points in $C_{\bar{n}}$ by aforementioned formulas, and denote the set of these points by

$$
\begin{aligned}
& \left\{\left(c_{k 1}, c_{k 2}, \ldots, c_{k \bar{n}}\right) \mid k=1 \sim q\right\} \\
& \quad=\left\{k\left(\gamma_{1}, \gamma_{2}, \ldots, y_{\bar{n}}\right) \mid k=1 \sim q\right\} .
\end{aligned}
$$

In simulations, the parameter value is taken as $p=2 \bar{n}+3$ and $q=5$.

(2) Generate $q$ uniformly distributed points in set $[l, u]$ by

$$
\begin{gathered}
B=\left\{\left(b_{k 1}, b_{k 2}, \ldots, b_{k \bar{n}}\right) \mid b_{k j}=l_{j}+c_{k j}\left(u_{j}-l_{j}\right),\right. \\
j=1 \sim \bar{n}, k=1 \sim q\} .
\end{gathered}
$$

Then, the points in $B$ are offspring of $X$ and $Y$.

3.2. Mutation Operator. Suppose that $X=\left(x_{1}, x_{2}, \ldots, x_{\bar{n}}\right)$ is any parent chosen for mutation. Let $X_{k}^{*}$ denote the best individual in current population $\operatorname{POP}(k)$ and let $X_{\text {best }}$ denote 
TABLE 3: The rates of return of 10 stocks for the first 30 days.

\begin{tabular}{|c|c|c|c|c|c|c|c|c|c|c|}
\hline Date & S1 & S2 & S3 & S4 & S5 & S6 & S7 & S8 & S9 & $\mathrm{S} 10$ \\
\hline 1 & -0.0050 & -0.0017 & 0 & 0.0013 & 0.0013 & 0.0039 & 0.0107 & -0.0073 & -0.0052 & 0.0210 \\
\hline 2 & -0.0188 & -0.0247 & -0.0056 & -0.0230 & -0.0166 & -0.0166 & -0.0045 & -0.0220 & -0.0183 & -0.0039 \\
\hline 3 & -0.0460 & -0.0219 & -0.0488 & -0.0370 & -0.0594 & -0.0444 & -0.0440 & -0.0141 & -0.0267 & -0.0299 \\
\hline 4 & -0.0067 & -0.0035 & -0.0141 & 0.0014 & -0.0055 & 0.0051 & 0.0080 & 0.0065 & 0.0027 & 0.0148 \\
\hline 5 & -0.0013 & 0.0112 & -0.0191 & -0.0217 & -0.0027 & -0.0072 & 0 & 0 & 0.0514 & -0.0159 \\
\hline 6 & 0.0054 & 0.0051 & -0.0182 & -0.0124 & -0.0166 & -0.0272 & -0.0235 & -0.0109 & 0.0026 & -0.0294 \\
\hline 7 & -0.0108 & 0 & 0.0062 & -0.0353 & -0.0240 & -0.0409 & -0.0323 & -0.0144 & -0.0026 & -0.0222 \\
\hline 8 & -0.0189 & -0.0186 & 0.0391 & -0.0044 & -0.0102 & -0.0298 & 0.0050 & -0.0123 & -0.0522 & 0.0014 \\
\hline 9 & -0.0014 & 0.0104 & 0.0107 & 0.0204 & 0.1051 & 0.0342 & 0.0217 & 0.0067 & 0.0164 & 0.0155 \\
\hline 10 & -0.0083 & -0.0043 & 0.0069 & 0.0029 & -0.0057 & 0.0066 & 0.0032 & 0.0011 & 0.0431 & 0.0069 \\
\hline 11 & 0.0028 & 0.0017 & -0.0105 & 0.0043 & 0 & -0.0022 & 0.0065 & 0 & -0.0129 & 0.0014 \\
\hline 12 & -0.0153 & -0.0181 & -0.0284 & -0.0199 & -0.0384 & -0.0185 & -0.0048 & 0 & -0.0210 & -0.0234 \\
\hline 13 & 0.0071 & -0.0157 & 0.0230 & -0.0220 & 0.0044 & -0.0078 & -0.0098 & -0.0101 & -0.0188 & -0.0042 \\
\hline 14 & 0.0126 & 0.0053 & 0.0141 & 0.0223 & 0.0282 & 0.0280 & 0.0163 & 0.0079 & 0.0162 & 0.0099 \\
\hline 15 & 0.0552 & 0.0379 & 0.0219 & 0.0507 & 0.0217 & 0.0286 & 0.0256 & 0.0155 & 0.0160 & 0.0237 \\
\hline 16 & -0.0170 & -0.0076 & -0.0091 & -0.0111 & 0.0181 & 0.0160 & -0.0016 & -0.0099 & -0.0079 & -0.0027 \\
\hline 17 & 0.0402 & -0.0034 & -0.0023 & 0.0181 & -0.0028 & 0.0347 & 0.0345 & 0.0122 & 0.0747 & 0.0261 \\
\hline 18 & -0.0182 & -0.0234 & -0.0208 & 0.0055 & -0.0234 & -0.0166 & -0.0137 & 0.0011 & -0.0274 & -0.0149 \\
\hline 19 & -0.0013 & 0.0096 & 0.0094 & -0.0123 & 0.0287 & -0.0124 & -0.0061 & -0.0055 & 0.0127 & -0.0027 \\
\hline 20 & -0.0130 & 0.0052 & -0.0012 & -0.0027 & -0.0027 & -0.0166 & 0.0093 & 0.0077 & 0 & 0.0014 \\
\hline 21 & -0.0213 & -0.0139 & 0.0012 & -0.0042 & -0.0167 & -0.0043 & -0.0046 & -0.0022 & -0.0101 & 0 \\
\hline 22 & 0.0041 & -0.0018 & 0.0153 & 0.0028 & 0.0196 & 0.0065 & 0.0156 & 0.0187 & 0.0408 & 0.0149 \\
\hline 23 & 0.0134 & 0.0167 & 0.0161 & 0.0235 & 0.0319 & 0.0244 & 0.0167 & 0.0065 & 0.0074 & 0.0162 \\
\hline 24 & 0.0053 & 0.0373 & -0.0011 & 0.0027 & -0.0081 & 0.0135 & 0.0015 & 0.0032 & -0.0024 & 0.0026 \\
\hline 25 & -0.0040 & -0.0051 & 0.0158 & 0.0108 & 0.0270 & 0.0185 & 0.0074 & 0.0331 & 0.0048 & 0.0132 \\
\hline 26 & -0.0146 & 0.0237 & -0.0156 & -0.0229 & -0.0283 & -0.0220 & -0.0103 & -0.0216 & 0.0024 & -0.0246 \\
\hline 27 & -0.0054 & -0.0116 & 0.0192 & 0.0136 & 0.0082 & 0.0320 & 0.0134 & 0.0211 & 0.0550 & 0.0728 \\
\hline 28 & 0 & -0.0091 & 0.0411 & 0.0054 & 0.0203 & -0.0111 & 0.0132 & 0.0093 & 0.0113 & 0.0272 \\
\hline 29 & -0.0136 & -0.0260 & -0.0150 & -0.0148 & -0.0013 & 0.0040 & 0 & -0.0010 & -0.0158 & 0.0331 \\
\hline 30 & 0.0138 & 0.0293 & 0.0163 & 0.0041 & -0.0109 & 0.0150 & -0.0044 & -0.0103 & 0.0572 & -0.0418 \\
\hline
\end{tabular}

the best individual obtained up to now. Then the offspring $O$ of $X=\left(x_{1}, x_{2}, \ldots, x_{\bar{n}}\right)$ by mutation is defined by

$$
\begin{gathered}
S=X+r_{1}\left(x_{k}^{*}-X\right), \\
O=S+r_{2}(k) \begin{cases}\left(X_{\text {best }}-S\right), & \text { if } S \text { is worse than } X_{\text {best }} \\
\left(S-X_{\text {best }}\right), & \text { otherwise, }\end{cases}
\end{gathered}
$$

where $r_{1}$ is a random number in $(0,1)$ and $r_{2}(k)=\exp (-c k)$ with $c$ being a random number in $(0,1)$.

\subsection{The Proposed Evolutionary Algorithm}

Algorithm 3. (1) (Initialization) Given population size $N$, crossover probability $P_{c}>0$ and mutation probability $P_{m}>0$. Randomly generate initial population POP (0). Let $k=0$.

(2) (Crossover) Randomly choose $\left[P_{c} \times N / 2\right]$ pairs of parents from POP $(k)$. For each pair, use Algorithm 2 to generate offspring. The set of all these offspring is denoted as $\mathrm{O}_{1}$.
(3) (Mutation) Randomly choose $\left[P_{m} \times\left|O_{1}\right|\right]$ parents from $O_{1}$. For each chosen individual $X$, use mutation operator to get an offspring $O$. The set of all these offspring is denoted as $\mathrm{O}_{2}$.

(4) (Selection) Select best [N/2] individuals among $\mathrm{POP}(k) \cup O_{1} \cup O_{2}$ to put into $\mathrm{POP}(k+1)$, and then randomly select $N-[N / 2]$ individuals among $\operatorname{POP}(k) \cup O_{1} \cup O_{2}$ to put into POP $(k+1)$. Let $k=k+1$.

(5) (Termination) If stop condition is satisfied, stop; otherwise, go to step (2).

\section{Experimental Results for Real Stock Market}

To evaluate the performance of the proposed optimization portfolio model based on a new risk measure called WES and the genetic algorithm, we conducted the experiments on randomly chosen ten stocks for 60 days (during January 2, 2014 to April 2, 2014) from Wind database (CFD) in Shenzhen Stock Exchange and the names and numbers of these ten stocks are shown in Table 1, where VKA represents A-shares of China Vanke Company Limited, PAB represents 
TABLE 4: The rates of return of 10 stocks for the last 30 days.

\begin{tabular}{|c|c|c|c|c|c|c|c|c|c|c|}
\hline Date & S1 & S2 & S3 & S4 & S5 & S6 & S7 & S8 & S9 & $\mathrm{S} 10$ \\
\hline 31 & -0.0068 & -0.0201 & -0.0053 & -0.0095 & -0.0312 & -0.0188 & 0 & -0.0104 & 0.0687 & -0.0171 \\
\hline 32 & -0.0219 & -0.0135 & 0.0579 & -0.0247 & -0.0192 & -0.0181 & -0.0205 & 0.0010 & -0.0348 & 0.0149 \\
\hline 33 & -0.0671 & -0.0384 & -0.0221 & -0.0394 & -0.0268 & -0.0265 & 0.0133 & -0.0011 & -0.0021 & 0.0233 \\
\hline 34 & -0.0179 & -0.0125 & -0.0384 & -0.0117 & -0.0249 & -0.0329 & -0.0444 & -0.0084 & -0.0611 & -0.0523 \\
\hline 35 & 0.0167 & 0.0082 & -0.0065 & 0 & 0.0074 & 0.0076 & 0.0077 & 0.0032 & 0.0045 & 0.0025 \\
\hline 36 & -0.0134 & 0.0117 & 0.0521 & -0.0192 & 0.0104 & -0.0214 & -0.0137 & -0.0084 & -0.0465 & -0.0288 \\
\hline 37 & 0.0197 & -0.0098 & -0.0093 & 0.0309 & 0.0338 & 0.0331 & 0.0248 & -0.0118 & 0.0331 & 0.0179 \\
\hline 38 & -0.0120 & -0.0082 & 0.0104 & 0.0177 & 0.0088 & 0.0293 & 0.0182 & -0.0022 & 0.0558 & 0.0216 \\
\hline 39 & 0.0400 & -0.0055 & -0.0010 & -0.0072 & 0.0058 & -0.0240 & -0.0015 & 0.0087 & 0.0128 & -0.0025 \\
\hline 40 & -0.0231 & -0.0257 & -0.0010 & -0.0014 & -0.0130 & -0.0160 & 0 & 0.0191 & -0.0170 & -0.0111 \\
\hline 41 & 0.0828 & 0.0065 & 0.0031 & 0.0391 & -0.0130 & 0.0238 & 0.0163 & 0.0201 & 0.0107 & -0.0051 \\
\hline 42 & -0.0068 & 0.0028 & 0.0051 & -0.0338 & -0.0098 & -0.0201 & 0.0015 & 0.0010 & 0.0401 & 0.0076 \\
\hline 43 & -0.0166 & -0.0458 & -0.0470 & -0.0333 & -0.0464 & -0.0435 & -0.0441 & 0.0225 & -0.0581 & -0.0303 \\
\hline 44 & 0.0487 & -0.0068 & 0.0076 & 0.0284 & 0.0015 & 0.0057 & 0.0046 & 0.0241 & 0.0260 & -0.0013 \\
\hline 45 & 0.0134 & 0.0107 & 0.0117 & 0.0015 & 0.0088 & 0.0022 & 0.0468 & 0 & -0.0259 & -0.0247 \\
\hline 46 & 0.0145 & 0.0200 & 0.0181 & 0.0115 & 0.0029 & 0.0011 & 0.1010 & 0.0047 & 0.0191 & 0.0397 \\
\hline 47 & -0.0234 & -0.0230 & 0.0052 & -0.0100 & -0.0158 & 0 & 0.0523 & -0.0773 & 0.0129 & 0.0270 \\
\hline 48 & 0.0303 & 0.0144 & 0.0533 & 0.0073 & 0.0205 & 0.0089 & 0.0485 & -0.0152 & 0.0190 & 0.0075 \\
\hline 49 & -0.0206 & -0.0086 & 0.0039 & -0.0130 & 0 & -0.0078 & -0.0221 & 0.0130 & -0.0020 & 0.0012 \\
\hline 50 & -0.0133 & -0.0135 & -0.0029 & -0.0015 & 0.0146 & 0.0169 & 0.0348 & -0.0268 & 0.0309 & 0.0111 \\
\hline 51 & 0.0013 & -0.0137 & -0.0176 & -0.0015 & -0.0307 & -0.0165 & -0.0181 & 0.0043 & -0.0101 & -0.0428 \\
\hline 52 & 0.0661 & 0.0685 & 0.0189 & 0.0681 & 0.0290 & 0.0324 & 0 & 0.0011 & 0 & 0.0636 \\
\hline 53 & 0.0062 & 0 & -0.0125 & 0.0384 & 0 & 0.0322 & 0.0576 & 0.0075 & -0.0201 & 0.0194 \\
\hline 54 & -0.0100 & -0.0176 & -0.0218 & 0.0159 & 0.0372 & -0.0031 & -0.0433 & 0.0011 & 0.0021 & -0.0178 \\
\hline 55 & -0.0163 & 0.0094 & 0.0091 & -0.0221 & -0.0052 & -0.0198 & -0.0024 & -0.0118 & -0.0124 & 0.0072 \\
\hline 56 & 0.0167 & 0.0066 & -0.0210 & 0.0601 & -0.0080 & 0.0362 & -0.0505 & 0.0216 & -0.0437 & 0.0216 \\
\hline 57 & 0.0177 & 0.0009 & -0.0163 & 0.0288 & 0.0013 & 0.0719 & -0.0389 & -0.0319 & -0.0370 & 0.0129 \\
\hline 58 & -0.0037 & -0.0009 & -0.0125 & -0.0388 & -0.0036 & -0.0473 & 0.0077 & -0.0306 & 0 & -0.0474 \\
\hline 59 & -0.0012 & 0.0028 & 0.0148 & -0.0209 & 0.0131 & 0.0350 & 0.0013 & 0.0282 & 0.0136 & -0.0036 \\
\hline 60 & 0.0258 & 0.0102 & 0.0010 & 0.0399 & 0.0052 & 0.0099 & 0.0118 & -0.0044 & 0 & 0.0157 \\
\hline
\end{tabular}

stock of Ping An Bank Company Limited, BLL represents stock of Baolilai Investment Company Limited, SZPRDA represents A-shares of Shenzhen Properties and Resources Development (Group) Limited, CSGA represents A-shares of CSG Holding Company Limited, SH represents stock of Shahe Industry, SZHA represents A-shares of Shenzhen Zhongheng Huafa Company Limited, SVOTA represents Ashares of Shenzhen Victor Onward Textile Industrial Company Limited, Konka A represents A-shares of Konka Group Company Limited, and SSIA represents A-shares of Shenzhen Shenbao Industrial Company Limited. We collect the daily returns and daily return rates from Shenzhen Stock Exchange in Wind database (CFD) in this period in the experiments.

In the experiments, the confidence level is chosen as $95 \%$ and the parameters in the proposed algorithm are taken as follows: $q=5, p=29, N=10, P_{c}=0.4$, and $P_{m}=0.1$. $n=10$ and $M=60$. We consider the case in which there is transaction cost of the risky asset for simplicity in the simulations; that is, let $k_{i}=0.0003$. We use the same values of parameters as those in [26]. That is, we suppose that investor only has currency initially; that is, $y_{i}^{0}=0, i=1 \sim n$. Thus, $y_{i}^{\prime \prime}=0$ and $y_{i}^{\prime}=y_{i}$ for $i=1 \sim n$. Let $t_{0}=t_{g}=0.00001$, $r_{n+1}=0.00007$, and $r_{p}=0.0016$. Also, we take weighted function $w(x)$ as follows: when $x \leq 0, w(x)=\exp (-\lambda x)$, and $w(x)=0$ when $x>0$, where $\lambda$ represents the risk aversion coefficient and $\lambda=0,0.1,1,5,10$.

We use the proposed algorithm to the optimization portfolio model (21). The results are given in Table 2 . The rates of return of the 10 selected stocks for 60 days are given in Tables 3 and 4.

It can be seen from Table 2 that, for four of five cases with different risk aversion coefficients, the risk values are very small (smaller than 0.05 ). Only for case $\lambda=10$, the risk value is relatively large (0.0933). Thus, in general speaking, we got very good portfolios.

Under the risk aversion coefficient of 0 , the optimal portfolio is $(0.1410,0.0000,0.0080,0.1103,0.0500,0.0805$, $0.0783,0.1433,0.0976$, and 0.2910 ), and the value of WES is 0.0252. This means that, in no risk aversion coefficient, the weighted expected Loss is less than 0.0252 . That is to say, there 
is a very small loss with no risk aversion coefficient. Similarly, under the risk aversion coefficient of 0.1 , the optimal portfolio is $(0.1045,0,0.0079,0.1107,0.0499,0.0808,0.0771,0.1431$, 0.0983 , and 0.2918 ), and the value of WES is 0.0256 . That is to say, under the risk aversion coefficient of 0.1 , the loss of portfolio is not more than 0.0256 . Next, under the risk aversion coefficient of 1 , the optimal portfolio is $(0.1272$, $0.0104,0.0384,0.1058,0.0261,0.0830,0.0931,0.1289,0.1046$, and 0.2823 ), and the value of WES is 0.0294 . In these three different risk aversion coefficient conditions, values of WESs are relatively small and performance of the portfolios in these cases is similar. Then, under the risk aversion coefficient of 5 , the optimal portfolio is $(0.1249,0.0079,0.0699,0.1208$, $0.0000,0.0750,0.0869,0.1278,0.1163$, and 0.2704$)$, and the value of WES is 0.0495 . That means that, under the risk aversion coefficient of 5, the loss of portfolio is a little bit larger but not more than 0.0495. Finally, under the risk aversion coefficient of 10 , the optimal portfolio is $(0.1266,0.0082$, $0.0833,0.1287,0,0.0660,0.0771,0.1255,0.1168$, and 0.2678$)$, and the value of WES is 0.0933 which is relatively larger.

It also can be seen from the experiments and Table 2 that when $\lambda$ is not too large (smaller than 5), the value of WES is relatively small and does not varied greatly. Thus when $\lambda$ varies in $[0,5)$, the values of WES vary only a little bit. That means that the proposed model is stable and the proposed algorithm is robust with the variations of parameter $\lambda$. However, when we take $\lambda$ values larger than 5 , especially larger than 10, the values of WES will be relatively large and vary greatly. This will result in the unstability of the model. Thus, for this model, we suggest that $\lambda$ is not taken to be larger than 5.

From the five different risk aversion coefficients, we can see that the value of WES is monotonely increasing with the increasing of the risk aversion coefficients. Thus, the bigger the risk aversion coefficients are, the more suitable the WES will be as a risk measure for conservative investors. In other words, by adjusting the risk aversion coefficients, the conservative investors would rather choose to concentrate their assets in a few stocks to get better benefits and control investment risks than to put their money to all the stocks to spread the investment risks.

\section{Conclusions}

Portfolio optimization model is very useful for the investors and stock companies, and setting up reasonable portfolio model is very important in practice. Based on this motivation, in this paper, a new portfolio optimization model by adopting a new risk measure [24] is proposed. This model is a nonlinear optimization model and is very difficult to be solved by using the existing optimization methods. To solve the model effectively, a new genetic algorithm based on uniform design method is proposed. To test the proposed model and algorithm, the simulations are made on 10 randomly chosen stocks for 60 days beginning from January 2, 2014 from Wind database (CFD) in Shenzhen Stock Exchange Markets. The results indicate that the proposed model is reasonable and the proposed algorithm is robust.

\section{Conflict of Interests}

The authors declare that there is no conflict of interests regarding the publication of this paper.

\section{Acknowledgment}

This work is supported by the National Natural Science Foundation of China (no. 71171158 and no. 61272119).

\section{References}

[1] H. M. Markowitz, "Portfolio selection," Journal of Finance, vol. 7, no. 1, pp. 77-91, 1952.

[2] W. J. Baumol, "An expected gain-confidence limit criterion for portfolio selection," Management Science, vol. 10, pp. 174-182, 1963.

[3] P. Jackson, D. J. Maude, and W. Perraudin, "Bank capital and value at risk," Journal of Derivatives, vol. 4, pp. 73-89, 1997.

[4] R. Fuss, D. G. Kaiser, and A. Zeno, "Value at risk, GARCH modelling and the forecasting of the hedge fund return volatility," Journal of Derivatives \& Hedge Funds, vol. 13, pp. 2-25, 2007.

[5] H. Rootzén and C. Klüppelberg, "A single number can’t hedge against economic catastrophes," Ambio, vol. 28, no. 6, pp. 550$555,1999$.

[6] P. Artzner, F. Delbaen, J. M. Eber et al., "Thinking coherently," Risk, vol. 10, pp. 33-49, 1997.

[7] P. Artzner, F. Delbaen, J.-M. Eber, and D. Heath, "Coherent measures of risk," Mathematical Finance, vol. 9, no. 3, pp. 203228, 1999.

[8] T. S. Beder, "VAR: seductive but dangerous," Financial Analysis Journal, vol. 9, pp. 12-24, 1995.

[9] C. Albanese, "Credit exposures, diversification risk, and coherent VaR," Tech. Rep., Department of Mathematics, University of Toronto, 1997.

[10] P. Carr, H. Geman, and D. B. Madan, "Pricing and hedging in incomplete markets," Journal of Financial Economics, vol. 62, no. 1, pp. 131-167, 2001.

[11] C. Acerbi, C. Nordio, and C. Sirtori, "Expected shortfall as a tool for financial risk management," http://arxiv.org/abs/condmat/0102304.

[12] R. T. Rockafellar and S. Uryasev, "Optimization of conditional value at risk," Journal of Risk, vol. 2, pp. 493-517, 2000.

[13] C. Acerbi, "Spectral measures of risk: a coherent representation of subjective risk aversion," Journal of Banking and Finance, vol. 26, no. 7, pp. 1505-1518, 2002.

[14] E. Rosazza Gianin, "Risk measures via $g$-expectations," Insurance: Mathematics and Economics, vol. 39, no. 1, pp. 19-34, 2006.

[15] Z. Chen and Y. Wang, "A new class of coherent risk measures based on p-norms and their applications," Applied Stochastic Models in Business and Industry, vol. 23, no. 1, pp. 49-62, 2007.

[16] Z. P. Chen and Y. Wang, "Two-sided coherent risk measures and their application in realistic portfolio optimization," Journal of Banking and Finance, vol. 32, no. 12, pp. 2667-2673, 2008.

[17] D. Chung and K. Hrazdil, "Liquidity and market efficiency: a large sample study," Journal of Banking and Finance, vol. 34, no. 10, pp. 2346-2357, 2010.

[18] A. Bosch-Domènech and J. Silvestre, "Reflections on gains and losses: a $2 \times 2 \times 7$ experiment," Journal of Risk and Uncertainty, vol. 33, no. 3, pp. 217-235, 2006. 
[19] R. T. Rockafellar, S. Uryasev, and M. Zabarankin, "Generalized deviations in risk analysis," Finance \& Stochastics, vol. 10, no. 1, pp. 51-74, 2006.

[20] J. Dhaene, M. J. Goovaerts, and R. Kaas, "Economic capital allocation derived from risk measures," North American Actuarial Journal, vol. 7, no. 2, pp. 44-59, 2003.

[21] S. Farinelli, M. Ferreira, D. Rossello, M. Thoeny, and L. Tibiletti, "Beyond Sharpe ratio: optimal asset allocation using different performance ratios," Journal of Banking and Finance, vol. 32, no. 10, pp. 2057-2063, 2008.

[22] H. Föllmer and A. Schied, "Convex measures of risk and trading constraints," Finance and Stochastics, vol. 6, no. 4, pp. 429-447, 2002.

[23] H.-J. Lüthi and J. Doege, "Convex risk measures for portfolio optimization and concepts of flexibility," Mathematical Programming, vol. 104, no. 2-3, pp. 541-559, 2005.

[24] Z. Chen and L. Yang, "Nonlinearly weighted convex risk measure and its application," Journal of Banking and Finance, vol. 35, no. 7, pp. 1777-1793, 2011.

[25] Z. Chen, L. Yang, D. Xu, and Q. Hu, "Tail nonlinearly transformed risk measure and its application," OR Spectrum, vol. 34, no. 4, pp. 817-860, 2012.

[26] L. Yang, Generalized convex risk measures and their applications in investment decision making [Ph.D. thesis], School of Mathematics and Statistics, Xi'an Jiaotong University, Xi'an, China, 2013.

[27] S. Das, A. Mukhopadhyay, A. Roy, A. Abraham, and B. K. Panigrahi, "Exploratory power of the harmony search algorithm: analysis and improvements for global numerical optimization," IEEE Transactions on Systems, Man, and Cybernetics, Part B: Cybernetics, vol. 41, no. 1, pp. 89-105, 2011.

[28] Y. Wang and C. Dang, "An evolutionary algorithm for global optimization based on level-set evolution and latin squares," IEEE Transactions on Evolutionary Computation, vol. 11, no. 5, pp. 579-595, 2007.

[29] Y. Wang, Y.-C. Jiao, and H. Li, "An evolutionary algorithm for solving nonlinear bilevel programming based on a new constraint-handling scheme," IEEE Transactions on Systems, Man and Cybernetics Part C: Applications and Reviews, vol. 35, no. 2, pp. 221-232, 2005.

[30] F. T. Fang and Y. Wang, Number-Theoretic Methods in Statistics, Chapman \& Hall, London, UK, 1994. 


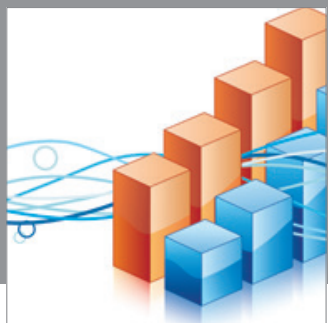

Advances in

Operations Research

mansans

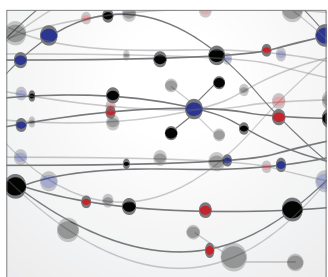

The Scientific World Journal
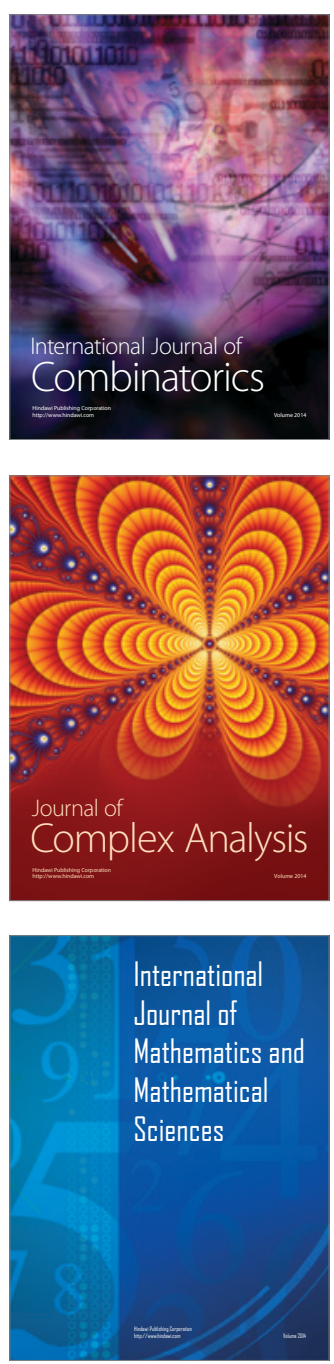
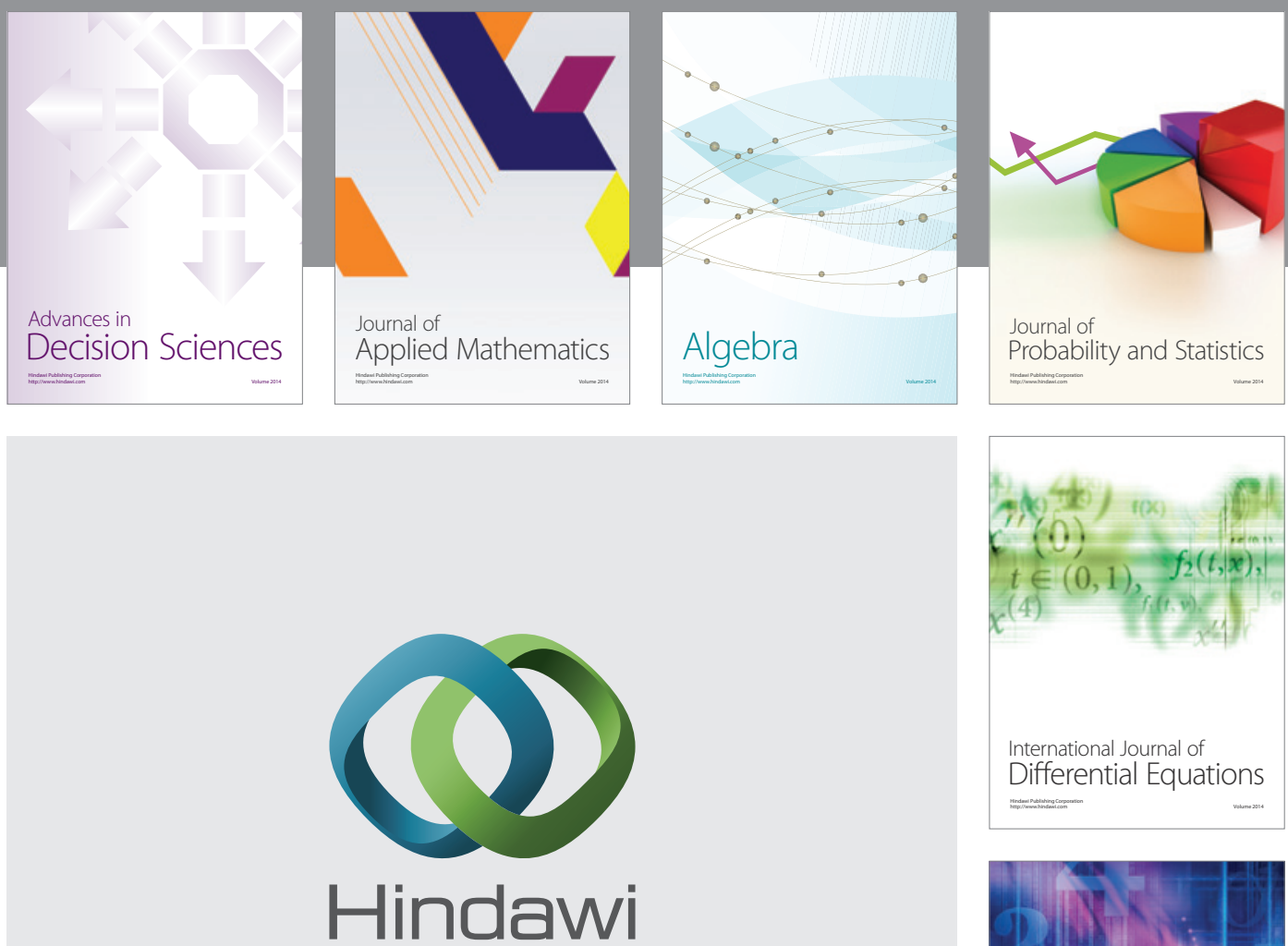

Submit your manuscripts at http://www.hindawi.com
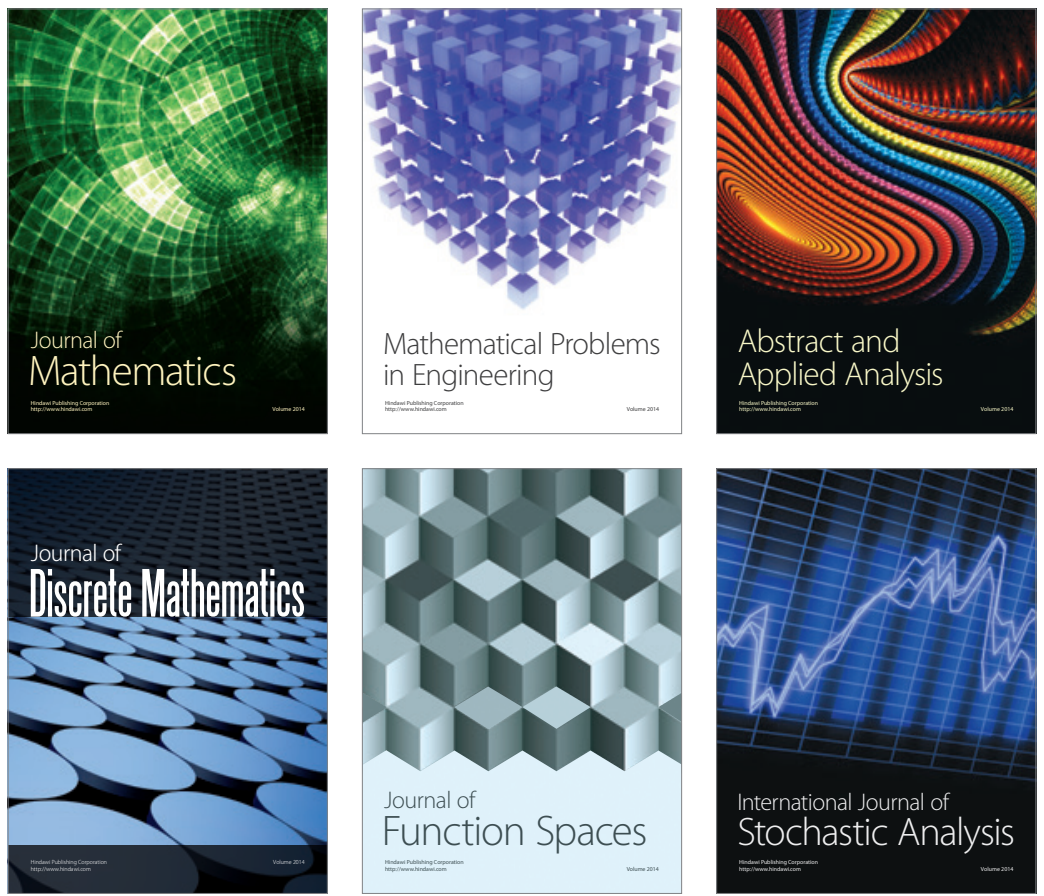

Journal of

Function Spaces

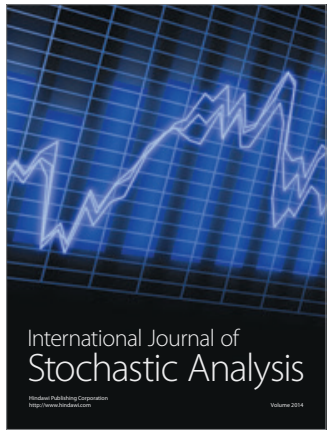

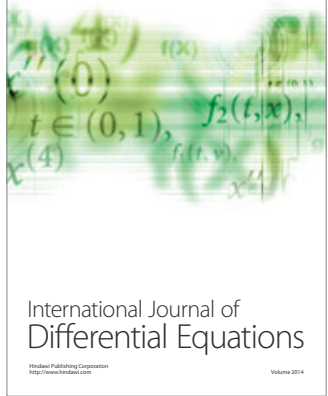
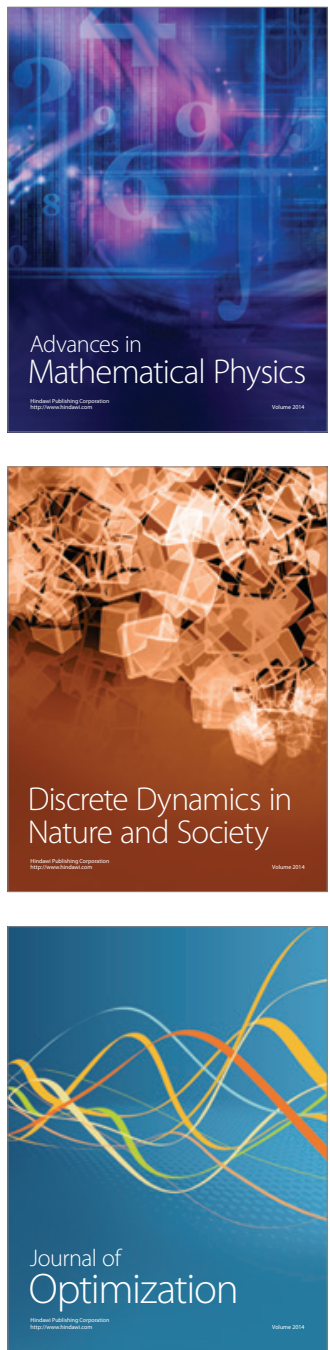